\section{Resistance Exercise Program During Breast Cancer Treatment: a Feasibility Study}

\section{Abstract}

Background: In the last two decades, physical training has been recognized as an important means of prevention and rehabilitation for patients with breast cancer. In Brazil, physical exercise for patients with breast cancer is a new area of study. We aimed to investigate the feasibility of implementing a resistance exercise program for patients undergoing breast cancer treatment and to examine the effects of the resistance exercise program on psychological and physical outcomes.

Methods: We conducted a non-randomized controlled trial. Eleven women (55.08 \pm 7.8 years) undergoing radiotherapy and hormone therapy for breast cancer were divided into exercise group $(n=6)$ and control group $(n=5)$. The feasibility was evaluated by training load performed by each patient compared to the total training load prescription. The resistance exercise program consisted of 12 weeks and 36 sessions ( $3 x$ per week), and intensity between 50 and $70 \%$ of one maximum repetition. Fitness (6-minute walk test), quality of life (EORTC QLQ-C30), mood states (BRUMS), sleep quality (Pittsburgh), and body image (Body Image after Breast Cancer) were investigated before and after resistance exercise program. Data were analyzed by comparison inter and intra groups $(p=0.05)$.

Findings: The resistance exercise program implementation was feasible ( $>80 \%)$. There were no adverse events due to the resistance exercise program, and the adherence averaged $83.6 \%$ with no dropouts. Twelve weeks of resistance exercise appeared to have positive effects on quality of life, sleep quality, physical fitness, body image, and fatigue in patients undergoing breast cancer treatment.

Conclusions: The implementation of a resistance exercise program in a university in the south of Brazil proved to be feasible, tolerable,
Claudia Arab ${ }^{1,2}$, Vitor Giatte Angarten', Tânia Brusque Crocetta1,2, Isabel Cristina Esposito Sorpreso ${ }^{2}$, Luiz Carlos de Abreu², Alexandro Andrade ${ }^{1}$

1 Laboratório de Psicologia do Esporte e do Exercício (LAPE). Universidade do Estado de Santa Catarina Florianópolis, Santa Catarina, Brasil.

2 Laboratório de Delineamento de Estudos e Escrita Científica da Faculdade de Medicina do ABC, Santo André, São Paulo, Brasil.

\section{Contact information:}

\section{Claudia Arab.}

Address: Programa de Pós-Graduação em Ciências do Movimento Humano. Centro de Ciências da Saúde e do Esporte. Universidade do Estado de Santa Catarina Florianópolis, Santa Catarina, Brasil.

झ ac.arabclaudia@gmail.com 
and adhered to breast cancer patients undergoing treatment. The 12week resistance exercise program apparently produces positive effects on psychological and physical outcomes in patients undergoing breast cancer treatment. This is the first study about physical training during breast cancer treatment in Brazil and seems to be a promising research area as well as internationally.

\section{Keywords}

Exercise; Breast Neoplasm; Feasibility Studies.

\section{Background}

Breast cancer (BC) is the most common and incident type of cancer in women worldwide and is the leading cause of cancer death in this population. Data from the National Cancer Institute showed a prediction of 54,120 new BC cases in Brazil in 2014 [1]. Cancer has an impact on many aspects of life and often results in numerous physical and psychological sequels in the short and long term [2]. As new BC therapies are developed and BC survivorship rates improve, physical activity behaviors and cardiovascular health will be increasingly important to maintain health among this population [3].

Despite the established efficacy of BC treatments (e.g. chemotherapy and radiation), they cause adverse effects on cardiovascular, metabolic, and quality-of-life outcomes of patients [4]. Some adverse effects can be acute, occurring primarily during treatment, whereas others may have a delayed onset and persist for years after the cessation of treatment [4]. Activities that were manageable before the disease start to cause pain and suffering to patients diagnosed with BC [5]. Consequently, the level of physical activity decreases and remains low for months, even after treatment. Decreased physical activity may be related to a higher mortality rate, lower quality of life, and increased risk of developing chronic diseases such as heart disease and diabetes $[8,9]$.

Efforts should be made to help BC patients in the diagnosis and treatment phases in order to provide a better quality of life and prognosis [8].
In the last two decades, physical training has been recognized as an important means of prevention and rehabilitation for patients with $B C$ [6]. The increased publications in this area proves the increase health professionals number interested in exploring alternative therapies with the potential to improve the overall health of $\mathrm{BC}$ patients while promoting relief of adverse effects of treatments [9]. Conducting research with cancer survivors presents many challenges with respect to recruiting and retaining participants [10]. The effects of different volumes and intensity of training must be explored, and exercise adherence rates need to be reported [10]. (Hanson et al., 2016).

In Brazil, physical training for patients with $\mathrm{BC}$ is a new area of study, and the application of a protocol of resistance exercise for BC patients undergoing treatment is an innovative practice. As proposed and directed by Timmerman et al. [11] before starting a randomized clinical trial, a study is necessary in order to model the methodology, including operations, to gain insight into the preliminary efficacy, revealing limitations and possible modifications to develop and continue the study. The objective of this study was to verify the implementation feasibility of a resistance exercise program for women undergoing treatment for BC. Secondly, we investigated the effects of resistance exercise program on fitness, quality of life, mood state, sleep quality, and body image in patients undergoing $B C$ treatment. 


\section{Methods}

\section{Study strategy}

This feasibility study was an experimental controlled non-randomized study and was approved by the Santa Catarina State University Ethics Committee, Santa Catarina, Brazil (493, 467/2013). The research was divulgated in hospitals and health centers, and the researchers made contacts with doctors and physiotherapists through pamphlets and meetings. Patients interested in participating in the study contacted the laboratory by telephone and scheduled an interview. They were informed about the research achievement, aims, relevance, and evaluation procedures. Those patients who agreed to participate and who met the inclusion criteria signed a written informed consent. The research was conducted at a university gym. The resistance exercise program feasibility was assessed by training load compared to the load that each patient was able to perform. The training load was calculated by: number of sessions $x$ exercises $x$ sets $x$ repetitions. The resistance exercise program was considered feasible if the training performed by each patient was higher than or equal to $80 \%$ training load.

\section{Study participants}

The inclusion criteria of study participants were women diagnosed with $B C$ in non-metastatic stages (0-III) who underwent surgical treatment. They had to present more than 80 points in the Karnofsky Performance Index and needed medical permission to engage in physical exercise program. All women were undergoing radiotherapy and/or hormone therapy during the study. Exclusion criteria were patients who had cardiopulmonary, metabolic, and/ or orthopedic disorders.

Eleven breast cancer patients participated in the study (55.08 \pm 7.8 years old). They were divided into exercise group $(E G)(n=6)$ and control group (CG) $(n=5)$. The EG patients had a mean age of $58( \pm 6.6)$ years, and the GC patients had a mean age of $54( \pm 7.9)$ years $(p=0.23)$. The patients who were not able to participate in the resistance exercise program (e.g., incompatible schedules and/ or distance from their home to the place of practice) were invited to compose the CG.

The EG patients were diagnosed with breast cancer about 23.5 ( \pm 16.4) months before the study and underwent surgical treatment for 11.67 ( \pm 12.6) months. In the CG, these values were $19.8( \pm 8.6)$ and 10.2 ( \pm 8.7) months, respectively. Patients of the EG had a primary breast cancer diagnosis and had mastectomy surgery without breast reconstruction (63.7\%). Most EG patients did not undergo post-surgical physiotherapy and were undergoing hormonal treatment; only two patients were in radiotherapy during the study. In the CG, one of the patients had recurrent breast cancer, most had mastectomy, and two underwent breast reconstruction. All GC patients underwent postoperative physiotherapy; three patients were on hormonal treatment and two were in radiotherapy during the study.

\section{Resistance exercise program}

We used part of the training protocol proposed by Arab et al. [12], which concerned to resistance exercise. The protocol is based on various studies and guidelines, such as Campbell et al. [13], American College of Sports Medicine roundtable on exercise guidelines for cancer survivors [14], Physical Activity Guidelines for Americans of the Department of Health and Human Services [15], Carayol et al. [16] metanalyses, Schmidt et al. [17], Battaglini et al [18], and Hwang et al [19].

The training program had duration of 12 weeks and 36 sessions that occurred three times per week on alternate days (Table 1). The sessions were structured in an initial part (5 to 10 minutes on a treadmill or stationary bike), a main part (40 minutes of strength physical exercise), and a final part (5 minutes of stretching major muscle groups).

The repetitions were planned in ranges, so the participants performed according to perceived 
Table 1. Structure of the Resistance exercise Program

\begin{tabular}{c|c|c|c|l|}
$\begin{array}{c}\text { Number } \\
\text { of } \\
\text { Sessions }\end{array}$ & $\begin{array}{c}\text { Intensity } \\
\%\end{array}$ & $\begin{array}{c}\text { Sets per } \\
\text { exercise }\end{array}$ & $\begin{array}{c}\text { Repetitions } \\
\text { per series }\end{array}$ & \multicolumn{1}{c}{ Exercises (n) } \\
\hline 12 & $50-60$ & 3 & $12-15$ & $\begin{array}{l}\text { Leg press, leg curl, seated cable row machine, supinated triceps bar and } \\
\text { abdominal (5) } \\
\text { Leg press, leg curl, seated cable row machine, supinated triceps bar, }\end{array}$ \\
\hline 12 & $55-65$ & 3 & $10-12$ & $\begin{array}{l}\text { Leg } \\
\text { dumbbell front raise, abdominal, and abdominal with no footrest (7) }\end{array}$ \\
\hline 6 & $55-65$ & 3 & $10-12$ & $\begin{array}{l}\text { Leg press, leg curl, leg extension, supinated triceps bar, pull down } \\
\text { machine, dumbbell lateral raises, abdominal and abdominal with no } \\
\text { footrest (8) }\end{array}$ \\
\hline 6 & $60-70$ & 3 & $8-10$ &
\end{tabular}

effort, which should be "mild to moderate" or when feeling "burning" pain. The minimum load training was 7,956 and a maximum of 9,720, so it was necessary that each participant got at least 6,365 (80\% load training).

The order of exercises performance was changed every two weeks. Initially, exercises were performed alternating body segment (exercises to upper limbs - UL x lower limbs - LL). After two weeks, the exercises were performed directly per body segment (all to $\mathrm{LL}$ and all for UL). The sets interval was one minute, and the intervals between exercises were about two minutes and controlled by the patients. The researchers recorded the loads according to the prescribed intensity in individual records for each patient. The patients recorded exercises, loads, sets and reps performed in each session.

The training sessions were conducted in groups and supervised by physical education and physiotherapy professionals at a university gym.

The proposed protocol was individually adjusted according to the needs and limitations of the patients. When patients could not perform the minimum required repetitions, the load was decreased. We used two methods for increasing load. The load was increased to the next level of the machinery or at least 5\% when patients underwent three series of the same exercise with the maximum required repetitions during three consecutive sessions. And when the patient could perform more repetitions than requested, the load was increased to $10 \%$ [12].
In cases of pauses or breaks in a training week, the load was regressed to the amount corresponding to two weeks above and thus correspondingly.

\section{Instruments}

Clinical and sociodemographic profiles were performed through our own recruitment records. The instruments used for patient evaluations were the 6-minute walk test (6MWT) [20], EORTC QLQ-C30 (European Organization for Research and Treatment of Cancer Quality of Life Questionnaire Core-30) [21], Brunel Mood Scale validated by Rohlfs et al. [22], Pittsburgh Sleep Quality Index translated and validated by Bertolazi [23], and Body Image After Breast Cancer Questionnaire translated and validated by Gonçalves [24]. The variables assessed were fitness, quality of life, mood state, sleep quality, and body image. Following the protocol of Brzycki [25], 1RM test was performed to predict the workload. In this test, the load is estimated by an equation that uses the load value used in a submaximal test, ideally up to 10 repetitions.

Assessments occurred before and after intervention by interview or self-administering the questionnaires individually in a room (except 6MWT, which was conducted on a running track), and took around an hour per participant. The load prediction test was carried out in the first exercise session, which was for learning the exercise techniques and becoming familiar with the equipment. 


\section{Statistical analysis}

Data were analyzed in Excel and statistical package SPSS version 20 using descriptive (mean, frequency, and standard deviation) and inferential statistics. Variables were treated according to data distribution using the Shapiro-Wilk test. The comparison of means of dependent variables (pre- and posttests) was performed by intra group test paired t-tests (parametric) and Wilcoxon (non-parametric), and for inter comparison the groups were applied the independent t-test and U Mann-Whitney tests. The significance level adopted was $\alpha=0.05$. Results with $p$ values between 0.05 and 0.07 were considered a trend towards significance, and we considered valid values when the effect size was $r \geq 0.5$.

\section{Results}

The training load average of the EG was 7,233.66 (991.43 SD), with a range of 6,241 and 8,668. The- refore, the resistance exercise program was feasible according to the $>80 \%$ training load criteria. The patients were able to maintain the proposed training intensity and loads. There were no adverse events arising from the practice of resistance exercise and no dropouts in EG patients. The EG adherence in the resistance exercise program was $83.6 \%$. Of the 36 planned sessions, the EG patients performed an average of 30 physical exercise sessions. After the resistance exercise program, the EG apparently improved fitness, functional quality of life, and sleep efficiency, and reduced levels of fatigue and physical limitations (body image). There was a tendency toward a reduction of mental confusion. In the CG, the pre- and post-tests showed an apparent decline in global health and a tendency to reduce muscular strength. There was no difference in other variables (Table 2).

The comparison between groups in the pre-test showed unfavorable differences in the EG in fatigue $(p=0.01)$, mental confusion $(p=0.01)$ and

Table 2. Psychological and physical outcomes pre and post resistance exercise program in exercise group and control group.

\begin{tabular}{|c|c|c|c|c|c|c|c|c|c|c|c|}
\hline & \multicolumn{5}{|c|}{ Exercise Group $(n=6)$} & \multicolumn{5}{|c|}{ Control Group $(n=5)$} \\
\hline & & $\begin{array}{l}\text { Pre Mean } \\
\text { (SD) }\end{array}$ & $\begin{array}{l}\text { Post Mean } \\
\text { (SD) }\end{array}$ & $\Delta$ & $\mathbf{P}$ & $r$ & $\begin{array}{l}\text { Pre Mean } \\
\text { (SD) }\end{array}$ & $\begin{array}{l}\text { Post Mean } \\
\text { (SD) }\end{array}$ & $\Delta$ & $\mathbf{P}$ & $r$ \\
\hline \multicolumn{2}{|c|}{ Fitness (m) } & $434.1(76)$ & $519.8(33.2)$ & 85.75 & $.02 *$ & .82 & $421.5(33.2)$ & $450.5(.7)$ & 29 & .427 & .78 \\
\hline \multirow{3}{*}{ ه̊ } & Global health & 75 (13.9) & $70.8(25.1)$ & -4.16 & .7 & .17 & $86.7(9.5)$ & $66.7(0)$ & -20 & $.01 *$ & .92 \\
\hline & Symptoms & $23.1(8.7)$ & $15.8(12)$ & -7.27 & .214 & .54 & $13.3(9.8)$ & $18.9(8.6)$ & 5.64 & .18 & .63 \\
\hline & Functional & $66.3(17.3)$ & $74.8(15.8)$ & 8,51 & $.028 *$ & .81 & $88.9(6.5)$ & $85.5(2.4)$ & -3.38 & .261 & .55 \\
\hline \multirow{6}{*}{ 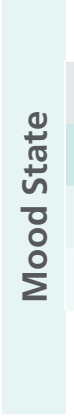 } & Tension & $7(4.6)$ & $4.8(4.4)$ & -2.17 & .157 & .60 & $2(2)$ & $2(2.1)$ & 0 & 1 & 0 \\
\hline & Depression & $6(5.8)$ & $2.8(3.4)$ & -3.17 & .276 & -.31 & $0.4(.9)$ & $1(1.2)$ & .6 & .414 & -.26 \\
\hline & Anger & $4.7(4.6)$ & $2.3(4.4)$ & -2.34 & .109 & -.46 & $0.2(.4)$ & $1(1.7)$ & .8 & .414 & -.26 \\
\hline & Vigor & $9.8(3.5)$ & $10.2(2.8)$ & .34 & .671 & -.12 & $10.8(1.6)$ & $5.8(3)$. & -5 & $.068+$ & -.58 \\
\hline & Fatigue & $7.2(2.8)$ & $3.3(2.3)$ & -3.84 & $.01 *$ & .88 & $1.6(3.1)$ & $.4(.9)$ & -1.2 & .284 & .53 \\
\hline & $\begin{array}{l}\text { Mental } \\
\text { Confusion }\end{array}$ & $6.3(2.7)$ & $3.8(4.4)$ & -2.5 & $.058+$ & -.55 & $1(1.2)$ & $1.2(1.3)$ & .2 & .705 & -.12 \\
\hline \multirow{3}{*}{$\begin{array}{l}0 \\
\frac{0}{0} \\
\frac{\sigma}{n}\end{array}$} & Global & $7(3.2)$ & $6(2.5)$ & -1.0 & .518 & .30 & $7.2(4.1)$ & $4.8(2.1)$ & -2.4 & .31 & .5 \\
\hline & Duration (h) & $7.2(1.5)$ & $7.7(.8)$ & .5 & .257 & -.33 & $7.4(.5)$ & $7.9(.5)$ & .5 & .102 & -.52 \\
\hline & Efficiency & $78(14.7)$ & 93.5 (13.3) & 15.47 & $.043^{*}$ & -.58 & $89.3(10.6)$ & $90.2(8.6)$ & .85 & 1 & 0 \\
\hline
\end{tabular}




\begin{tabular}{|c|c|c|c|c|c|c|c|c|c|c|c|}
\hline & \multicolumn{5}{|c|}{ Exercise Group $(n=6)$} & \multicolumn{5}{|c|}{ Control Group $(n=5)$} \\
\hline & & $\begin{array}{l}\text { Pre Mean } \\
\text { (SD) }\end{array}$ & $\begin{array}{l}\text { Post Mean } \\
\text { (SD) }\end{array}$ & $\Delta$ & $\mathbf{P}$ & $r$ & $\begin{array}{l}\text { Pre Mean } \\
\text { (SD) }\end{array}$ & $\begin{array}{l}\text { Post Mean } \\
\text { (SD) }\end{array}$ & $\Delta$ & $\mathbf{P}$ & $r$ \\
\hline \multirow{6}{*}{ 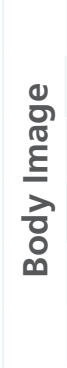 } & Vulnerability & $21.3(11)$ & $19.2(5.8)$ & -2.16 & .413 & .37 & $20.4(3.8)$ & $20.2(4.1)$ & -.2 & .928 & .05 \\
\hline & Body stigma & 44.3 (13.4) & $35.8(12.5)$ & -8.5 & .137 & .62 & $37.4(6.1)$ & $38(2.1)$ & .6 & .812 & .13 \\
\hline & Limitations & $16.3(3.7)$ & $13.3(3.3)$ & -3 & $.042 *$ & -.59 & $13.4(1.5)$ & $15(2)$ & 1.6 & .221 & -.39 \\
\hline & Body Concern & $16.5(5.7)$ & $14.3(5)$ & -2.17 & .249 & .50 & $16.4(4.8)$ & $16.8(4)$ & .4 & .833 & .11 \\
\hline & Transparency & $13(6.7)$ & $11.7(5.7)$ & -1.33 & .297 & .46 & $8.4(2.1)$ & $9(3.3)$ & .6 & .766 & .16 \\
\hline & Arm Concern & 6.8 (3.3) & $5.2(2.8)$ & -1.66 & .236 & -.34 & $6.6(.5)$ & $6(1.2)$ & -.6 & .317 & -.32 \\
\hline
\end{tabular}

${ }^{\star} \mathrm{P}<0.05 ;+0.05>\mathrm{p}<0.07 ; \mathrm{SD}$ : standard deviation; $\Delta$ : pre and post-test means difference; $r$ : effect size; $r$ bold: large effect size; m: meters; QoL: quality of life; Q: quality; h: hours

functional quality of life ( $p=0.023)$. The post-test showed favorable differences in the EG in fatigue $(p=0.021)$, vigor $(p=0.024)$, and fitness $(p=$ 0.002).

\section{Discussion}

The resistance exercise program for $B C$ patients undergoing treatment has proven to be feasible and tolerable, with a high adherence rate and no adverse effects or dropouts. The resistance exercise program apparently caused positive effects on fitness, functional quality of life, sleep quality, body image, and fatigue levels, avoiding negative impacts on quality of life and possibly on muscular strength and mental confusion. Our study presents limitations. First, the small sample size. We had difficulty in sample recruitment, because no hospitals or health services collaborated with the study and there were participation exercise program barriers, e.g. distance between the university and residences of the patients and exercises sessions scheduled just during the morning. Therefore, we could not randomized study participants. During the study, the patients reported unwanted events, such as familiar death, which may influence the results and were not associated with physical exercise practice. Although the participants showed high adherence to the resistance exercise program, there was no desistence and they demonstrated motivation during workouts.

BC patients can present various adverse effects from the treatment and disease. Penttinen et al. [26] found that after neoadjuvant therapies, patients presented depression (25.7\%), fatigue $(20.4 \%)$, and depression and fatigue (14\%), with impairments in quality of life and worse sleep quality than non-depressed and non-fatigued patients. Patients with untreated depression presented amplified physical symptoms of BC treatment, which can cause greater disability and poor treatment adherence [27]. Cancer patients commonly present physical problems such as reduced physical capacity, muscular strength, endurance, and physical function, and consequently decreased levels of physical activity [28]. The total expended energy in different types and intensities of occupational physical activity and physical exercises is associated with better physical function and general health of BC patients [29]. BC patients undergoing treatment need to improve the physical and mental aspects that allow performing usual activities, reduced pain and suffering $[5,6]$. In addition, physical inactivity is directly associated with morbidity. Reduced physical fitness is the main risk factor of mortality in the normal population and cardiovascular disease [30]. Regular physical activity is beneficial in terms of total mortality [5] and is 
inversely associated with greater pain and weight gain in BC survivors [31].

Development of actions for $\mathrm{BC}$ patients is necessary for better diagnosis and treatment coping in order to provide a better quality of life and prognosis [8]. Studies have shown that physical exercise for cancer patients has been feasible [32] safety, adhered and with increases in psychological and physiological parameters [9]. Fairman et al [4] conducted a systematic review to evaluate the comparable efficacy of exercise as a supportive care intervention during different primary breast cancer treatments. The findings demonstrated that exercise is a safe, well-tolerated lifestyle interventions that can attenuate many of the adverse effects accompanying treatment, and yield significant, clinically meaningful improvements in select fitness, physiological, and patient-reported outcomes in BC patients who are undergoing different treatment [4]. In the posttest, EG related less limitations and more physical competences and skills, as well as better body image and functional quality of life. Therefore, the EG presented higher sleep efficiency $(78 \%$ in the pretest to $94 \%$ in the post-test, which means more time lying in bed actually asleep) and decreased fatigue, which is one of the most frequent adverse and stressful events of cancer treatment [33]. Retention rates averaged $86 \%$ [4], similar as our results. The resistance exercise program proposed in our study proved to be a viable and possibly effective action.

In Brazil, since 2011 the Ministry of Health has emphasized actions to control cardiovascular disease, diabetes, cancer, and chronic respiratory disease predominantly through smoking control, physical inactivity, unhealthy diet, and harmful use of alcohol [22]. However, there are no governmental guidelines recommending physical activity for the Brazilian population as a strategy of health promotion. Physical activity is the easiest and most viable and available way to good health, and the evidence of its benefits, the physical inactivity levels are still high [34]. Exercise interventions utilizing strength training have great potential to impact the lives of cancer survivors, by helping to increase both quality and duration of life, particularly if exercise programs can expand to include effective home and communitybased protocols that can be delivered on a large scale [10].

Ortega and Fernandéz [35] and De Luca et al [36] pilot studies investigated the effect of concurrent aerobic and strength training in $\mathrm{BC}$ survivors. The exercise programs were proposed for 22 and 24 weeks, respectively. The results were positive. Both studies showed significant improvement in oxygen consumption, strength, and improvements on patient's quality of life $[35,36]$. The positive effects of a combined aerobic and strength training program in breast cancer survivors highlight the importance of the early inclusion of structured physical activity in the rehabilitation protocol [36]. Travier et al [37] suggest the inclusion of exercise intervention in the daily clinical practice starting early during adjuvant treatment. This multi-centre controlled trial randomly implemented 18-week supervised exercise intervention during chemotherapy and it showed to be feasible and safe. Moreover, BC patients could decrease physical fatigue and reduce diminishment of cardiorespiratory fitness and improve muscle strength [37].

Our 12-week resistance exercise program showed to be a promising potential therapy to complement BC treatment. The results show that strength training is potentially therapeutic and could be used as a supplementary treatment strategy, such as for reducing the use of painkillers, muscle relaxants, and mood regulators. Corroborating to the feasibility study of Timmerman et al. [11], the number of participants in our study was a limitation, and the effects we found may be more significant in a larger sample.

The implementation of a resistance exercise program proved to be feasible, tolerable, and adhered to by breast cancer patients undergoing treatment. The 12-week resistance exercise program apparently 
produces positive effects on fitness, quality of life, fatigue, sleep quality, and body image in patients undergoing breast cancer treatment. Further studies with larger samples are needed. This is the first study about physical exercise during breast cancer treatment in Brazil and seems to be a promising research area as well as internationally. Institutions in Brazil could adopt this low-cost practice with apparently high effectiveness in order to contribute to the $\mathrm{BC}$ population.

\section{Acknowledgments}

We are thankful for what UDESC, and the students Patrícia Morgana Rentz Keil, Clara Knierim Correia, Vanessa Lima, and Beatriz Bebiano and all study participants have supported in study realization.

\section{Funding \\ CAPES}

\section{Competing and Conflicting Interests}

None

\section{References}

1. Facina T. Estimate of Cancer Incidence in 2014-Brasil2014. Available on: http://www.inca.gov.br/estimativa/2014/index.asp

2. Hacker E. Exercise and quality of life: strengthening the connections. Clin J Oncol Nurs. 2009; 13(1): 31-9.

3. Arem H, Sorkin M, Cartmel B, Fiellin M, Capozza S, Harrigan M, Ercolano E, Zhou Y, Sanft T, Gross C, Schmitz K. Exercise adherence in a randomized trial of exercise on aromatase inhibitor arthralgias in breast cancer survivors: the Hormones and Physical Exercise (HOPE) study. J Cancer Surviv. 2016; 1-9.

4. Fairman CM, Focht BC, Lucas AR, Lustberg MB. Effects of exercise interventions during different treatments in breast cancer. J Community Support Oncol. 2016; 14(5): 200-209.

5. Larsson IL, Jönsson C, Olsson AC, Gard G, Johansson K. Women's experience of physical activity Following breast cancer treatment. Scand J Caring Sci. 2008; 22(3): 422-29.

6. Volaklis KA, Halle M, Tokmakidis SP. Exercise in the prevention and rehabilitation of breast cancer. Wien Klin Wochenschr. 2013; 125(11-12): 297-301.
7. Huy C, Schmidt ME, Vrieling A, Chang-Claude J, Steindorf K. Physical activity in the German breast cancer patient cohort: One-year trends and characteristics associated with change in activity level. Eur J Cancer. 2012; 48 (3): 297-304.

8. Olympio PCdAP, Amorim MHC, Lima EdFA. Stress and immune response in women with mastectomies during treatment with tamoxifen. Rev. enferm. UERJ. 2012; 20 (1): 15-20.

9. Battaglini $C L$, Mills $C R$, BL Phillips, Lee JT, Story $C E$, Nascimento MGB et al. Twenty-five years of research on the effects of exercise training in breast cancer survivors: A systematic review of the literature. J Clin Oncol World. 2014; 5 (2): 177-90.

10. Hanson ED, Wagoner CW, Anderson T, Battaglini CL. The Independent Effects of Strength Training in Cancer Survivors: a Systematic Review. Curr Oncol Rep. 2016; 18(5): 1-18.

11. Timmerman $\mathrm{H}$, de Groot JF, Hulzebos HJ, de Knikker R, Kerkkamp $H E$, van Meeteren NL. Feasibility preliminary effectiveness of preoperative and therapeutic exercise in patients with cancer: a pragmatic study. Physiother Theory Pract. 2011; 27 (2): 117-24.

12. Arab C, Andrade, Keil PMR, Crocetta TB. Physical Exercises for Women in Treatment of Breast Cancer. Rev Conexão UEPG. 2014; 10 (2): 224-37.

13. Campbell KL, Neil SE, Winters-Stone KMW. Review of exercise studies in breast cancer survivors: attention to principles of exercise training. Br J Sports Med. 2012; 46: 909-916.

14. PANEL Expert. American College of Sports Medicine roundtable on exercise guidelines for cancer survivors. J ACSM. 2010; 14091426.

15. US Department of Health and Human Services, and US Department of Health and Human Services. Physical activity guidelines for Americans. 2008.

16. Carayol M, Bernard P, Boiche J, Riou F, Mercier B, CoussonGélie F, Romain AJ, Delpierre C, Ninot G. Psychological effect of exercise in women with breast cancer receiving adjuvante therapy: what is the optimal dose needed?. Ann Oncol. 2013; 24: 291-300

17. Schmidt ME, Chang-Claude J, Vrieling A, Seibold P, Heinz J, Obi N, Flesch-Janys D, Steindorf K. Association of pre-diagnosis physical activity with recurrence and mortality among women with breast cancer. Int J Cancer. 2013; 133 (6): 1431-40

18. Battaglini CL, Mihalik JP, Bottaro M, Dennehy C, Petschauer MA, Hairston LS, Shields EW. Effect of exercise on the caloric intake of breast cancer patients undergoing treatment. B J Med Biol Res. 2008; 41: 709-715.

19. Hwang JH, Chang HJ, Shim YH, Park WH, Park W, Huh SJ, Yang $\mathrm{JH}$. Effects of Supervised Exercise Therapy in Patients Receiving Radiotherapy for Breast Cancer. Yonsei Med J. 2008; 49 (3): 443-450.

20. AT Society. ATS Statement: Guidelines for the Six-Minute Walk Test. Am J Respir Crit Care Med. 2002; 166(1): 111-7. 
21. Aaronson NK, Ahmedzai $S$, Bergman B, Bullinger $M$, Cull $A$, Duez NJ, et al. The European Organization for Research and Treatment of Cancer QLQ-C30: a quality-of-life instrument for use in international clinical trials in oncology. J Natl Cancer Inst. 1993; 85 (5): 365-76.

22. Rohlfs ICPdM, Rotta TM, Luft CBD, Andrade $A$, Krebs RJ, Carvalho Td. The Brunel Mood Scale (BRUMS): tool for early detection of the overtraining syndrome. Rev Bras Med Sport. 2008; 14 (3): 176-81.

23. Bertolazi AN, Fagondes SC, Hoff LS, Dartora EG, Miozzo IC, de Barba ME et al. Validation of the Brazilian portuguese version of the Pittsburgh sleep quality index. Sleep Med. 2011; 12 (1): 70-5.

24. Gonçalves CdO, Tavares MdCGC, Campana ANNB, Cabello C. Validation of the instrument "Body image after breast cancer" in Brazil. Motriz: Rev. educ. fis. 2014; 20 (1): 8-

25. Brzycki M. Strength testing-predicting a one-rep max from reps-to-fatigue. J. phys. educ. recreat. Dance. 1993; 64 (1): 8890.

26. Penttinen $H$, Saarto $T$, Kellokumpu-Lehtinen $P$, Blomqvist $C$, Huovinen $\mathrm{R}$, Kautiainen $\mathrm{H}$, et al. Quality of life and physical performance and activity of breast cancer patients after adjuvant treatments. Psychooncology. 2011; 20 (11): 1211-20.

27. Fann JR, Thomas AM, Katon WJ, Cowley D, Pepping $M$, McGregor BA, et al. Major depression after breast cancer: a review of epidemiology and treatment. Gen Hosp Psychiatry. 2008; 30(2): 112-26

28. van Weert E, Hoekstra-Weebers JE, May AM, Korstjens I, Ros WJ, van der Schans CP. The development of an evidence-based physical self-management rehabilitation program for cancer survivors. Patient Educ Couns. 2008; 71 (2): 169-90.

29. Basen-Engquist K, Hughes D, Perkins H, Shinn E, Taylor DC. Dimensions of physical activity and their relationship to physical and emotional symptoms in breast cancer survivors. Surviv J Cancer. 2008; 2 (4): 253-61.

30. Duncan BB, Chor D, Aquino MLE, Bensenor IM, Mill JG, Schmidt $\mathrm{MI}$, et al. Chronic noncommunicable diseases in Brazil: priority for confrontation and research. Rev Saúde Pública. 2012; 46 (Suppl 1): 126-34

31. Forsythe LP, Alfano CM, George SM, McTiernan A, Baumgartner $K B$, Bernstein $L$, et al. Pain in long-term breast cancer survivors: the role of body mass index, physical activity, and sedentary behavior. Breast Cancer Res Treat. 2013; 137 (2): 617-30.

32. Quist M, Rørth M, Langer S, Jones LW, Laursen JH, Pappot $H$, et al. Safety and feasibility of a combined exercise intervention for inoperable lung cancer patients undergoing chemotherapy: a pilot study. Lung Cancer. 2012; 75 (2): 203-8.

33. Arnold ME, Taylor NF. Exercise for patients with cancer: reducing disease-related fatigue. Future Oncol. 2011; 7 (2): 165-7.

34. Sebastião E, Schwingel A, Chodzko-Zajko W. Brazilian physical activity guidelines as a strategy for health promotion. Rev Saúde Pública. 2014; 48(4): 709-12.
35. Ortega JAF, Fernandéz JAP. Effects of a combined strength and high-intensity aerobic exercise program in breast cancer survivors: A pilot study. Apunts. Medicina de l'Esport. 2016; 51 (189): 3-12.

36. De Luca V, Minganti C, Borrione P, Grazioli E, Cerulli C, Guerra E, Bonifacino A, Parisi A. Effects of concurrent aerobic and strength training on breast cancer survivors: a pilot study. Public health. 2016

37. Travier N, Velthuis MJ, Bisschop CNS, van den Buijs B, Monninkhof EM, Backx F, Los M, Erdkamp F, Bloemendal HJ, Rodenhuis C, de Roos MAJ, Verhaar M, Huinink DB, van der Wall E, Peeters PHM, May AM. Effects of an 18-week exercise programme started early during breast cancer treatment: a randomised controlled trial. BMC medicine. 2015; 13(1): 1
Publish in International Archives of Medicine

International Archives of Medicine is an open access journal publishing articles encompassing all aspects of medical science and clinical practice. IAM is considered a megajournal with independent sections on all areas of medicine. IAM is a really international journal with authors and board members from all around the world. The journal is widely indexed and classified Q1 in category Medicine. 